\title{
Research and Design on 17 DF Humanoid Robots
}

\author{
Yanfang Wang ${ }^{1, a}$, Yilun Yang ${ }^{2, b}$ and Zhijun $\mathrm{Li}^{3, \mathrm{c}}$ \\ School of Automation, Wuhan University of Technology, Wuhan, Hubei, 430070, China \\ ayfwangzdh@whut.edu.cn, ${ }^{\mathrm{b}}$ ylyang@whut.edu.cn, ${ }^{\mathrm{c}}$ jzhli@whut.edu.cn
}

\begin{abstract}
Keywords: Servo Control, Tracking, Microcontroller, PWM signal.
Abstract. This paper mainly discussed a multi-degree-of-freedom, multi-servo-control methods in walking process of a bipedal robot, 4-way multi-infrared tracking and other related content. A robot designed according to the relevant principles can automatic tracking, collect the signal through the transmitting and receiving tube, and converts the signal which is identifiable for the microcontroller into digital signal. Controlled by the microcontroller, the robot can accomplish straight line moving, turning, stopping, waving, bowing and other actions and adjustments during walking routes.
\end{abstract}

\section{Introduction}

With the constant development of the society, and the constant improvement of the science and technology, people hope to create a requirement which can replace human to do some high-risk or high-precision works. In this situation, the discipline about robots has born. In 1959, the first robot in this world was born; it has been more than 50 years up till the present moment. Since the 21 th century, the rapid development of the computer science and technology has enhanced the comprehensive promotion of the robotics. At present, the robot has been developed to the third generation, with recognition, reasoning, planning and intelligent learning mechanism, it can combine perception with action intelligently, and can work in an unstable environment [1].

In other countries, the technology of the industrial robots has gradually matured, and the US robotics has been a leading position all the time, their robots have reliable property, full function and high accuracy, the intelligent technology has been developed very fast, and has been widely used in the aerospace, automotive and other fields. In China, the robot industry started late, but the growth momentum is very strong, a full range of industrial robots have been developed in the last ten years, and been widely used [1]. For now, our country should start with the point of production and application; combine with China's national conditions to speed up the production of the practical robots which have simple structure and low-costs. And the basic requirement for the intelligent robots is whether it can identify the road automatically and complete the related tasks.

A tracking robot was designed based on the STC12C5A60S2 microcontroller; the robot totally has 17 joints. One degree of freedom in the head, 6 degrees of freedom in the arms, and 10 degrees of freedom in the legs. It requires that the robot starts from the scratch line, and walk along the black guide lines, at the side of the lines placed obstacles. In the process of moving, the robot can detect obstacles and adjust the routes itself to avoid obstacles. When reaching the final point, the robot can wave its hands and make a bow for gratitude.

\section{The hardware design of the control system}

The robot consists of two parts, mechanical carrier and control system, the mechanical carrier uses 17 LD-2015 digital biaxial servos which are designed for robots, this kind of servo has high twisting force, high speed, low noise, small virtual spaces and dead areas, it can twist 360 degrees when power failure, and it can be precisely rotated 180 degrees when power on controlled, the rotation angle is controlled by a microcontroller. This paper mainly introduced the part of the control system, and the design is divided into the following three modules: the drive unit module, servo drive module, tracking and the obstacle avoidance module. 
In order to make the robot flexibly to carry a variety of sensors, the bottom of the drive unit was separated from the upper platform gait algorithm. Because of each joint on the robot are driven by servos, the drive for 17 servos was special designed as the underlying drive unit to drive the joints of the robot. Upper platform was designed to send the parameters of each joint to the bottom drive unit in real time through a universal synchronous / asynchronous receiver transmitter (USART). System block diagram shown in Fig. 1.

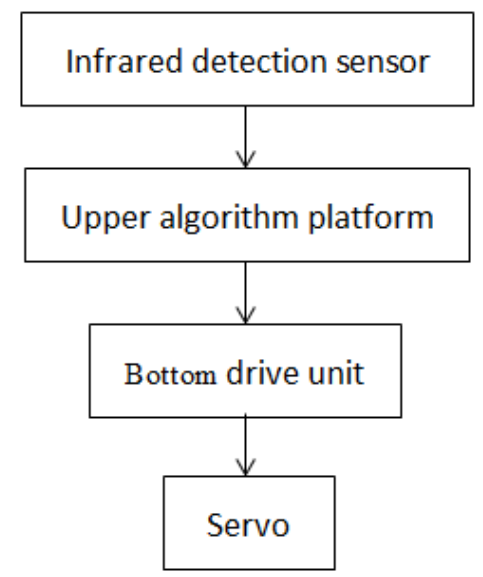

Figure 1 System block diagram

Servo is a kind of drive which servos by position, the range of rotation cannot exceed 180 degrees, mainly composed by the following components: helm, reduction gears, position feedback potentiometer, DC motor, control circuit boards and so on. The block diagram of the servo's working principle as shown in Fig. 2, there is a reference circuit inside the servo, it can product a reference signal which period is $20 \mathrm{~ms}$, and which width is $1.5 \mathrm{~ms}[2]$, and has a comparator. The control circuit board controls the motor rotates by receiving a control signal from the signal line, the motor drives the reduction gear and moves a linear potentiometer for position detection, the control circuit compares the feedback voltage with the input control pulse signal, the result drives the DC motor rotates forward or backward, to make the output position of the gear matches with the expected value.

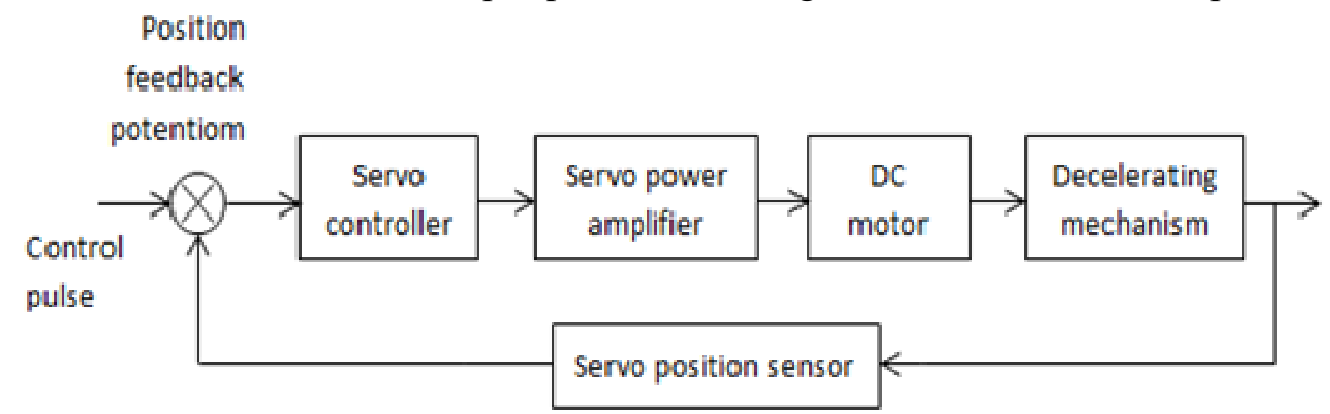

Figure 2 the block diagram of the servo's working principle

The control signal of the servo is the pulse width modulation (PWM) signal which period is $20 \mathrm{~ms}$; it's width changes from $0.5 \mathrm{~ms}$ to $2.5 \mathrm{~ms}$, corresponding to the position of the rudder plate from 0 to 180 degrees, changes linearly. That is, to provide it with a certain width, it's output shaft will remain in the corresponding degree, no matter what the external torque change, it will not change the output angle to the new corresponding position until it was given an additional pulse width.

In view of the above characteristics of the servo, the AVR microcontroller and multi-level control PC software were specially selected to jointly control the rotation of the servos. The AVR microcontroller was developed by the ATMEL, which has built-in Flash and high speed, according to the variable duty cycle PWM wave generated by its internal timer / counter, when it received the signal from the control module, AVR microcontroller generates the corresponding PWM pulse to control the servo rotates corresponding degrees to achieve the movement of the joints. 
Microcontroller STC12C5A60S2 is used as servo control module in this system, which contains a new 8051 core of high-speed, low power consumption, super anti-jamming. The instruction code is fully compatible with the traditional 8051 ,.but its speed is 8-12 times faster than the traditional one. It internals integrated MAX810 special reset circuit; two-way PWM; 8-way high-speed 10 A / D conversion, the speed can reach to $250 \mathrm{KHz}(250 \mathrm{~K} / \mathrm{S})$.It mainly used to motor control, strong interference occasions.

This microcontroller has dual serial ports. I use serial port 2 (RXD2 / TXD2) to connect with AVR microcontroller in this design. The planned gait parameters are stored in STC12C5A60S2.Then the MCU will send the appropriate data to the underlying joint drive unit, which the information is generated by a timer clock. At the same time, sensors detect environmental signals, and sent to the microcontroller. According to these information, MCU timely adjustments to the state of motion.

Robot tracking and obstacle avoidance uses an 8-way infrared detection tracking avoidance module. The infrared transmitting and receiving tubes act as probe. The core of the control circuit uses the LM339 voltage comparator devices. Infrared sensors are mounted on the robot square feet less than the height at $10 \mathrm{~mm}$.

The principle of detection pavement black line: 8 pairs of infrared tube (model ST L88) are installed in parallel robot leg; connect with LM324 to build 8 road of integrated operational amplifier circuit. It exports TTL level, and the distance to the road around $8 \mathrm{~mm}$. The infrared emission tube emits light to the road, if it is white, infrared light is reflected, receiving tube receives the reflected light, and then through the output amplifier circuit low level 0 to I/O port of MCU. On the contrary, when the infrared light is absorbed by the black lines meet, receive tube does not receive the reflected light. It outputs high level 1 to the I/O port of the MCU. Get the number of level through the I/O port; the MCU makes the corresponding instruction, to control the robot to turn left or right to adjust the direction of walking.

\section{The software design of the control system}

The theme of the design software is the detection of the black line and the obstacle going in the same time to control the direction when the robot walks, to accomplish the task based on the detection of the various sensors.

The main program was shown in Fig.3. Eight groups of infrared tube mounted side by side on the top of the robot's foot, four for most middle class 1 direction controller, perimeter 4 for two directional controllers.

If SENSOR1 or SENSOR2 detected the black line that the robot's foot pressed on the left part of the black line, it should be controlled to walk left in front, or if SENSOR3 SENSOR4 detect the black line that the robot foot pressed on the right part of the black line, it should be controlled to walk right in front, after reorientation, the robot continues to walk along with the straight line, and kept repeating the operation to detect the black line (Fig. 4). Level 2 was prepared for the first level, the steering efforts between the two levels must cooperate with each other, generally, level 2 will play a role until it beyond the control of the level 1 , so its rotational force is greater than level 1 , the size can be achieved by changing the PWM duty cycle. 


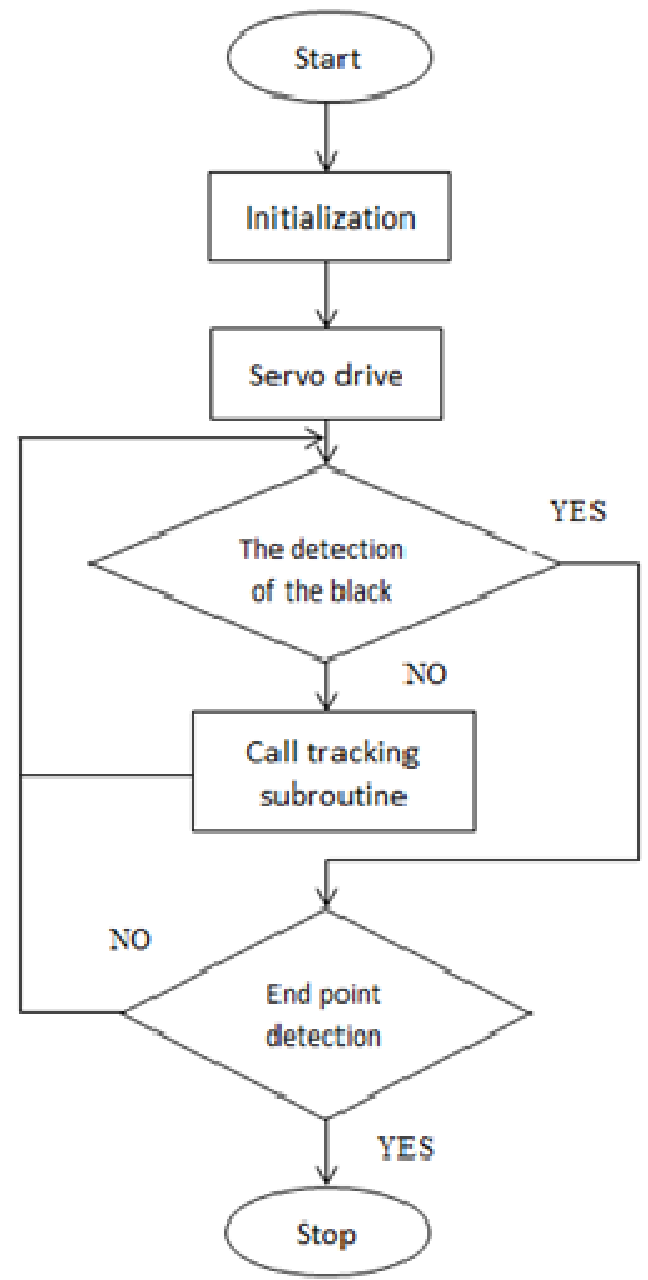

Figure 3 the main program flow chart

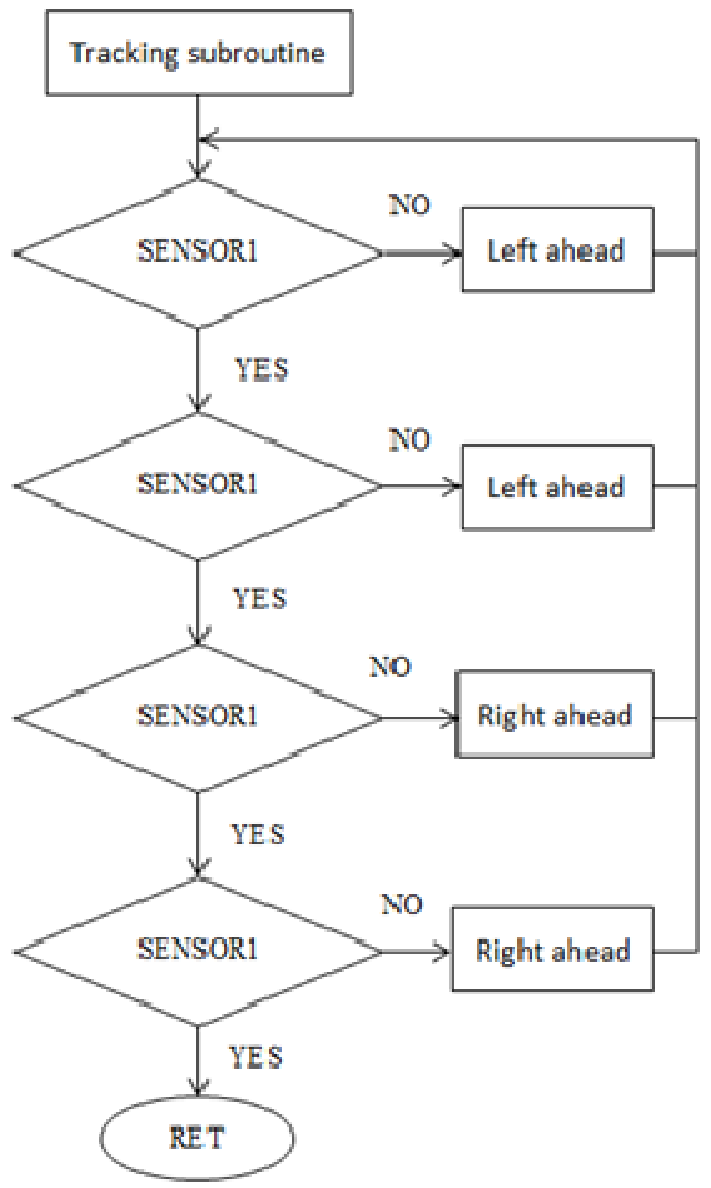

Figure 4 tracking subroutine flow chart

The record of the experiments

\begin{tabular}{|c|c|c|}
\hline Sequence Number & Sensor & Results \\
\hline 1 & sensor 2 on the left foot failed & slight shift to the left \\
\hline 2 & sensor 1and2 on the left foot failed & significant shift to the left \\
\hline 3 & sensor 2 on the right foot failed & slight shift to the right \\
\hline 4 & sensor 1 and2 on the right foot failed & significant shift to the right \\
\hline 5 & all sensors failed & not move along the black line \\
\hline 6 & all sensors bright & move along the black line \\
\hline
\end{tabular}

\section{Literature References}

References are cited in the text just by square brackets [1]. (If square brackets are not available, slashes may be used instead, e.g. /2/.) Two or more references at a time may be put in one set of brackets $[3,4]$. The references are to be numbered in the order in which they are cited in the text and are to be listed at the end of the contribution under a heading References, see our example below.

\section{Conclusions}

In actual production, the robot is small, the power is a key issue, in the consideration of the cost and performance and so on, a dedicated $7.4 \mathrm{~V}$ lithium polymer rechargeable battery was chose, due to the normal operating voltage of the servo is $6-7.4 \mathrm{~V}$, so the large buck chip is necessary to supply power. Experiments show that the servo is stable, has good performance in following, and easy to adjust 
speed, the robot walks stably, has high frequency pace, and large stride, the program has a strong versatility. By using the servo drive to control the robot to achieve the planned gait is a simple and effective method.

\section{Acknowledgement}

In this paper, the research was supported by Nature Science and Technology Major Project of China (2011ZX4002-21).

\section{References}

[1] ZHANG Tuo, Dai Ya-wen: Mechanical \& Electrical Engineering Technology, Vol. 38(2009)

[2] QIN Ping: Value Engineering, Vol. 32(2013), p. 62

[3] LIU Gequn, LU Jingchao, YAN Jianguo: Machinery \& Electronics, Vol. 2(2004), p. 76

[4] LIU Xue-jingn: Journal of Tianjin Vocational Institutes, Vol. 13(2011), p. 82

[5] FANG Qing-shan, LIN Chun-fang: Small \& Special Electrical Machines, Vol. 7(2009), p. 56

[6] FENG Xiao-wei, WANG Lei-yang, LI Zheng-sheng: Modern Electronics Technique, Vol. 34(2011), p. 167

[7] REN Zhi-min: Techniques of Automation and Applications, Vol. 27(2008), p. 85

[8] FU Li, LIU Wei-guo, YI Qiang: Small \& Special Electrical Machines, Vol. 2(2006), p. 28

[9] Zhou Yonglong, Lei Jinkui: Computer Measurement \& Control, Vol. 19(2011), p. 66 